\title{
Vorschläge für eine engere Zusammenarbeit zwischen OSZE und Europarat
}

\author{
Frank Evers, André Härtel, Marietta König"
}

\section{Zusammenfassung}

Der Europarat und die OSZE unterstützen beide die Einhaltung von menschenrechtlichen, rechtsstaatlichen und demokratischen Standards sowie die Stabilität in Europa. Beide Organisationen sind damit von der nachlassenden Bereitschaft zur multilateralen Kooperation sowie der zunehmenden Verletzung von Normen betroffen. Dieser Beitrag formuliert Vorschläge für eine engere Zusammenarbeit zwischen dem Europarat und der OSZE. Regierungen sollten mehr politische, personelle, und finanzielle Unterstützung leisten. Die Kommunikation auf der politischen Ebene sollte durch eine Stärkung der Europarat-OSZE Koordinierungsgruppe und die Wiederbelebung der "2+2"-Treffen auf Führungsebene intensiviert werden. Weiterhin sollten die externen Büros und Feldoperationen beider Organisationen enger zusammenarbeiten und dafür die nötigen Ressourcen erhalten. Schließlich sollten der Europarat und die OSZE ihre Politik- und Rechtsberatung so weit wie möglich miteinander abstimmen.

\section{Schlagworte}

OSZE, Europarat, Europäische Union, internationale Organisationen, Feldoperationen

Bitte zitieren als: Frank Evers, André Härtel, Marietta König, Vorschläge für eine engere Zusammenarbeit zwischen OSZE und Europarat, OSCE Insights 9 (Baden-Baden: Nomos, 2021), unter: https://doi.org/10.5771/9783748911630-09

\section{Einleitung ${ }^{1}$}

Der Europarat (Council of Europe, CoE) und die OSZE unterstützen die Einhal-

* Dr. Frank Evers

Institut für Friedensforschung und Sicherheitspolitik an der Universität Hamburg (IFSH)

evers@ifsh.de

Dr. André Härtel

Institut für Friedensforschung und Sicherheitspolitik an der Universität Hamburg (IFSH)

haertel@ifsh.de tung von Menschenrechten, Grundfreiheiten, Demokratie und Rechtsstaatlichkeit in Europa und können auf eine langjährige Zusammenarbeit zurückblicken. Beide Organisationen sind von der zu-

\footnotetext{
Marietta S. König

Büro der Generalsekretärin, OSZESekretariat

Marietta.Koenig@gmx.de

Bitte beachten: Dieser Beitrag basiert auf den persönlichen Ansichten der Autoren und spiegelt nicht unbedingt die offizielle Position des Europarats, der OSZE oder ihrer Mitglieds- und Teilnehmerstaaten wider.
} 
nehmenden Verletzung von Normen, dem schwindenden Vertrauen in demokratische Institutionen und einer Zunahme der politischen Radikalisierung in ihren Mitglieds- bzw. Teilnehmerstaaten betroffen. Eine engere Zusammenarbeit zwischen dem Europarat und der OSZE wäre hilfreich, um diesen Entwicklungen entgegenzuwirken. Allerdings setzen sich die Mitglieds- und Teilnehmerstaaten trotz wiederholter Diskussionen zu wenig hierfür ein.

Dieser OSCE Insights-Beitrag verfolgt zwei Ziele: den Stand der Zusammenarbeit zwischen dem Europarat und der OSZE zu analysieren und nach Wegen zu suchen, die Kooperation zu intensivieren. Die Zusammenarbeit auf der Arbeitsebene sollte verbessert und durch mehr Interaktion auf Führungsebene ergänzt werden. Letzteres gilt insbesondere für die Europarat-OSZE-Koordinierungsgruppe, die zu formalisiert ist. Auch eine Wiederbelebung der sog. "2+2"-Treffen auf Führungsebene wäre hilfreich. Das gleiche gilt für eine engere Zusammenarbeit in jenen Ländern, in denen beide Organisationen mit ihren Feldoperationen bzw. externen Büros präsent sind. Wir empfehlen dem Europarat und der OSZE darüber hinaus eine engere Abstimmung ihrer Politik- und Rechtsberatung. Vor allem die größeren EU-Mitgliedsstaaten sollten in beiden Organisationen auf solche Verbesserungen hinwirken.

Für diesen Beitrag wurden zwischen September und Dezember 2017 Interviews mit Vertreter*innen der beiden Organisationen in Den Haag, Straßburg, Warschau und Wien sowie mehrere Anschlussgespräche im Jahr 2020 geführt.
Auch basiert dieser Beitrag auf der Analyse der Dokumente, die die Zusammenarbeit zwischen beiden Organisationen regeln.

Der erste Abschnitt untersucht den Status Quo der Zusammenarbeit zwischen der OSZE und dem Europarat. Der zweite Abschnitt beschreibt die politischen und strukturellen Hindernisse einer engeren Zusammenarbeit. Der dritte und letzte Abschnitt enthält Handlungsempfehlungen für Regierungen und die Exekutivorgane der beiden Organisationen, wie diese Zusammenarbeit vertieft werden könnte.

\section{Die Interaktion zwischen OSZE und Europarat}

Der Europarat und die OSZE fördern beide Sicherheit und Stabilität in Europa, nehmen diese Aufgabe aber unterschiedlich wahr. Die OSZE ist die weltweit größte regionale Sicherheitsorganisation im Sinne von Kapitel VIII der Charta der Vereinten Nationen (UN) und bietet ein Forum für politische Verhandlungen. Die 57 Teilnehmerstaaten erörtern regelmäßig Sicherheitsfragen im Ständigen Rat und im Forum für Sicherheitskooperation. Die OSZE ist die größte Organisation für Konfliktmanagement in Europa und verfügt auch über ein umfangreiches Netzwerk von Feldoperationen.

Der Europarat zählt 47 Mitgliedsstaaten. Er trägt zur Stabilität in Europa bei, indem er für Menschenrechte, Demokratie und Rechtsstaatlichkeit eintritt, insbesondere durch die Verabschiedung von eigenen Rechtsnormen sowie die 
Mitwirkung bei der Umsetzung internationaler Abkommen und Konventionen. Eine maßgebliche Bedeutung kommt dabei der Europäischen Konvention zum Schutz der Menschenrechte und Grundfreiheiten (EMRK, 1950) zu. Aber auch die mehr als 220 anderen Abkommen und internationalen Verträge des Europarats tragen zusammen mit dem umfangreichen Apparat für ihre Umsetzung zum Schutz der Rechte der in den Mitgliedsstaaten lebenden Bürger*innen bei. Die Rechtsinstrumente des Europarats sowie die normsetzenden politischen Verpflichtungen der OSZE und ihre starke Feldpräsenz ergänzen sich. ${ }^{2}$

Zwar arbeiten beide Organisationen seit langem zusammen. Bei verschiedenen Gelegenheiten haben sie auch den "flexiblen und pragmatischen Charakter" 3 ihrer Zusammenarbeit betont sowie ihre grundlegende Absicht, sich „gegenseitig zu ergänzen und zu stärken “. ${ }^{4}$ Die Zusammenarbeit ist insbesondere auf der Arbeitsebene eng. Sie beruht auf Komplementarität, Transparenz, demokratischer Verantwortung und dem gegenseitigen Respekt für Mandat, Mitgliedschaft und Autonomie der jeweils anderen Organisation. Auf Führungsebene behindert eine stark ritualisierte Kommunikation aber ein engeres Zusammenwirken. Spielraum für Verbesserungen gibt es ebenfalls im Feld.

\section{Bestehende Kooperationsformate}

Flexibilität und Pragmatismus sind die Leitprinzipien der vier Schlüsseldokumente, die die Zusammenarbeit zwischen dem Europarat und der OSZE regeln. Ziel ist, Dopplungen zu vermeiden und die jeweiligen Stärken bestmöglich zu nutzen.

Dokumente, die die Zusammenarbeit zwischen Europarat und OSZE regeln

1. CoE/OSCE, Relations Between the Council of Europe and the OSCE: Common Catalogue of Co-operation Modalities, SEC.GAL/30/00, 4 April 2000 [OSCE], CoE Secretariat, $\mathrm{CM}(2000) 52,25$ April 2000 [CoE].

2. OSCE, Enhanced Co-operation Between the Organization for Security and Co-operation in Europe (OSCE) and the Council of Europe (CoE), Permanent Council Decision PC.DEC/637, 2 December 2004.

3. OSCE, Co-operation Between the Organization for Security and Cooperation in Europe (OSCE) and the Council of Europe, Permanent Council Decision PC.DEC/670, 28 April 2005.

4. CoE/OSCE, Declaration on Co-operation Between the Organization for Security and Co-operation in Europe and the Council of Europe, 17 May 2005.

Diese Dokumente führen eine Reihe von Formaten für Zusammenarbeit auf:

- die gegenseitige Entsendung von Vertreter*innen zu Sitzungen der Entscheidungsgremien und parlamentarischen Versammlungen sowie die Einrichtung von Verbindungsbüros; 
- die Koordinierungsgruppe, die $2004^{5}$ als reguläres Sitzungsformat auf höchster Ebene eingerichtet wurde, um die Zusammenarbeit innerhalb von vier formell vereinbarten Themenbereichen zu erörtern;

- hochrangige "2+2"-Treffen des amtierenden Vorsitzenden und des Vorsitzenden des Ministerkomitees, der beiden Generalsekretäre sowie der Leiter von Institutionen und Führungskräfte;

- "2+2"-Treffen auf Führungsebene, Treffen von Parlamentariern, gemeinsame Sitzungen unter Beteiligung von nationalen Expert*innen und Vertreter"innen der Sekretariate (beide "2+2"-Formate sind derzeit inaktiv, können jedoch aktiviert werden, s. Empfehlungen unten);

- hochrangige Dreiertreffen zwischen den Vorsitzenden und Generalsekretären der OSZE und des Europarats sowie dem Generaldirektor des Büros der Vereinten Nationen in Genf und anderen Repräsentanten (jährlich von 1993 bis 2011); ${ }^{6}$

- Zusammenarbeit zwischen Europarats- und OSZE-Institutionen in den vier formell vereinbarten Themenbereichen;

- Sekretariatskooperation und Informationsaustausch;

- Ad-hoc-Kontakte und Konsultationen, einschließlich Besprechungen auf Arbeitsebene. ${ }^{7}$

Daneben gab es im Laufe der Jahre eine Reihe von Arbeitsvereinbarungen, Absichtserklärungen und Korrespondenzen zwischen beiden Organisationen, um die Interaktion zusätzlich zu fördern. Ein Beispiel ist der Briefwechsel im November 2019 zwischen der damaligen Direktorin des OSZE-Büros für Demokratische Institutionen und Menschenrechte (ODIHR) und der Generalsekretärin des Europarats zu Bereichen der Zusammenarbeit (siehe unten).

Die Zusammenarbeit ist je nach Ebene mehr oder weniger intensiv: sie ist spärlich und unflexibel auf der höheren politischen Ebene (namentlich in der Koordinierungsgruppe), intensiv auf der Arbeitsebene zwischen den Institutionen und ungleichmäßig im Feld.

\section{Unflexible Kommunikation auf höchster Ebene}

Die Zusammenarbeit zwischen dem Europarat und der OSZE auf politischer Ebene stellt eine besondere Herausforderung dar. Die 2004 eingerichtete Koordinierungsgruppe ist das offizielle Format für hochrangige Treffen zwischen beiden Organisationen. Die Gruppe tritt zweimal im Jahr abwechselnd in Straßburg oder Wien zusammen. Am 13. November 2020 tagte sie zum insgesamt 32. Mal und aufgrund von COVID-19-Beschränkungen zum zweiten Mal online. Einige Beobachter*innen interpretieren die ursprüngliche Vereinbarung, sich „nach Bedarf und mindestens alle sechs Monate zu treffen", 8 als Verpflichtung zu intensiverer Kommunikation. Abhängig vom jeweiligen thematischen Schwerpunkt umfasst die Teilnehmerliste seitens des Europarats den Vorsitzenden und das Büro der Ministerbeauftragten, den Vorsitzen- 
den der Berichterstattergruppe für Auswärtige Beziehungen und Vertreter*innen des Sekretariats. Die Liste der OSZETeilnehmer umfasst Vertreter*innen der Troika, des Sekretariats, darunter das Büro des Sonderbeauftragten und Koordinators für die Bekämpfung des Menschenhandels, und Vertreter*innen der Institutionen, insbesondere den Hohen Kommissar für Nationale Minderheiten (HKNM) und das ODIHR.

Die Tagesordnung der Treffen der Gruppe ist stark formalisiert, die Zusammenarbeit auf vier Aufgabenkreise festgelegt: (a) die Bekämpfung des Terrorismus, (b) die Bekämpfung des Menschenhandels, (c) die Förderung von Toleranz und Nicht-Diskriminierung und (d) der Schutz der Rechte von Personen, die nationalen Minderheiten angehören. ${ }^{9}$ Diese strikten Vorgaben lassen wenig Spielraum. Ursprünglich waren die vier Bereiche nur als ein Ausgangspunkt gedacht, allerdings wurden sie nie erweitert. Mittlerweile herrscht unter den Teilnehmerstaaten große Uneinigkeit über weitere Themenbereiche; selbst innerhalb der vier existierenden Themenbereiche, beispielsweise bei Minderheitenproblemen, Toleranz und Nichtdiskriminierung sowie Gender-Mainstreaming, bestehen starke Differenzen.

\section{Flexible Interaktion auf Arbeitsebene}

Die operative Zusammenarbeit ist weniger formalisiert und zudem viel weiter entwickelt als im Falle der Koordinierungsgruppe. So schätzen hochrangige Mitarbeiter*innen beider Organisationen die hervorragenden Beziehungen auf dieser Ebene. Es besteht ein hohes Maß an Kenntnis der jeweiligen Partnerinstitutionen, vor allem zwischen dem ODIHR und der Europäischen Kommission für Demokratie durch Recht (besser bekannt als Venedig-Kommission). Dabei folgt die gemeinsame Arbeit etablierten Verfahren. Mehrere OSZE-Feldoperationen tauschen regelmäßig Informationen mit dem Europarat aus und organisieren gemeinsame Veranstaltungen und Projekte. Allerdings finden sich derlei gemeinsame Initiativen und Veranstaltungen im Feld sowie damit verbundene Themen selten in den Sitzungen der Koordinierungsgruppe oder in Berichten an die Mitglieds- bzw. Teilnehmerstaaten wieder.

Das ODIHR ist die federführende Institution für gemeinsame Wahlbeobachtungsmissionen mit der Parlamentarischen Versammlung des Europarats (PACE) und der Parlamentarischen Versammlung der OSZE. Wahlbeobachter*innen bewerten die Zusammenarbeit zwischen den beiden Versammlungen (sowie dem Europäischen Parlament und von Zeit zu Zeit der Parlamentarischen Versammlung der NATO und dem Kongress der Gemeinden und Regionen des Europarats) als ausgezeichnet. Zudem ist die Zusammenarbeit bei der Wahlbeobachtung auch in vielen Ländern für die breite Öffentlichkeit sichtbar. Gemeinsame Wahlbeobachtungsmissionen sind daher ein Flaggschiff der Kooperation.

Konsultationen gibt es darüber hinaus zwischen dem ODIHR und der Menschenrechtskommissarin des Europarats, die regelmäßiger Gast in OSZE-Foren 
ist. Konstruktive Beziehungen bestehen auch zwischen den OSZE-Institutionen, dem Kongress der Gemeinden und Regionen des Europarats, der Konferenz der Internationalen Nichtregierungsorganisationen und dem Europäischen Gerichtshof für Menschenrechte (EGMR). Mitarbeiter*innen des Europarats heben Praktiken hervor wie die Einladung hochrangiger OSZE-Vertreter*innen zu Sitzungen, die Teilnahme der OSZE an den Lenkungsausschüssen des Europarats und ihren nachgeordneten Gremien sowie die Interaktion mit den Feldoperationen der OSZE. ${ }^{10}$

Hinzu kommt, dass beide Organisationen Dokumente wie Entscheidungen, Urteile und Richtlinien der jeweils anderen Organisation nutzen und Texte gemeinsam erarbeiten. Insbesondere die Venedig-Kommission und das ODIHR haben aufgrund einer Kooperationsvereinbarung eine lange Tradition der gemeinsamen Erarbeitung von Stellungnahmen und Leitlinien, beispielsweise zur Religionsfreiheit oder zur Versammlungs- und Vereinigungsfreiheit. ${ }^{11}$ Gerade in denjenigen Bereichen, für die gemeinsame Richtlinien erarbeitet wurden, ist die $\mathrm{Zu}$ sammenarbeit intensiv.

Das ODIHR arbeitet nicht nur mit der Venedig-Kommission bei der Überprüfung von Rechtsvorschriften und Stellungnahmen und mit der Parlamentarischen Versammlung des Europarats anlässlich von Wahlen zusammen. Daneben besteht für Themen von beiderseitigem Interesse auch mit dem Sekretariat des Europarats eine Kooperation. Beispiele für gemeinsame Themen sind die Förderung von Toleranz und Nichtdiskrimi- nierung sowie der Schutz der Menschenrechte (einschließlich der Rechte von Minderheiten wie etwa der Roma und Sinti). Konkret arbeiten das ODIHR und das Sekretariat des Europarats unter anderem bei der Unterstützung von Menschenrechtsverteidigern und bei Maßnahmen gegen Hassverbrechen zusammen. Experten*innen beider Institutionen tauschen Informationen und Berichte aus und besprechen diese bei gemeinsamen Treffen. Wenn sich Möglichkeiten hierfür bieten, leiten sie auch gemeinsame Initiativen in die Wege. Das ODIHR verwendet die Länderberichte und politischen Empfehlungen der Europäischen Kommission gegen Rassismus und Intoleranz (ECRI) für den Aufbau eigener Kapazitäten, während das ECRI die ODIHR-Daten zu Hassverbrechen in seinen Länderberichten verwertet. Auch der Beratende Ausschuss für das Rahmenübereinkommen zum Schutz nationaler Minderheiten (FCNM) verweist in seinen Länderberichten häufig auf Daten zur Hasskriminalität des ODIHR sowie auf die thematischen Empfehlungen und Leitlinien des HKNM. Der HKNM verwendet die Stellungnahmen des Beratenden Ausschusses zum FCNM und die Berichte des Sachverständigenausschusses der Europäischen Charta der Regionaloder Minderheitensprachen. Hinzu kommen Besuche des HKNM zur Erörterung länderbezogener Minderheitenfragen mit hochrangigen Vertreter*innen in Straßburg und Venedig.

Ein Briefwechsel zwischen der ODIHR-Direktorin Ingibjörg Sólrún Gísladóttir und der Europarats-Generalsekretärin Marija Pejčinović Burić im No- 
vember 2019 diente dem Zweck, die jeweiligen Expert"innenteams auf jene Bereiche aufmerksam zu machen, in denen eine Zusammenarbeit zwischen ODIHR und dem Europarat existiert. ${ }^{12}$ Ähnliches könnte auch zwischen dem Europarat und dem HKNM oder dem Beauftragten für Medienfreiheit (RFoM) in Betracht gezogen werden.

\section{Ungleichmäßige Kooperation im Feld}

Interaktion im Feld ist ein Eckpfeiler der Zusammenarbeit zwischen Europarat und der OSZE. Beide Organisationen verfügen über ein großes Netzwerk von auswärtigen Büros beziehungsweise Feldoperationen. Der Europarat unterhält externe Büros in 17 Mitgliedsstaaten, vier Verbindungsbüros sowie ein Büro in $\mathrm{Pa}$ ris. Standorte befinden sich unter anderem in Ländern wie der Türkei oder den drei Südkaukasusstaaten, in denen die OSZE nicht oder nicht dauerhaft präsent ist. Mit Stand von 2020 unterhält die OSZE 16 Feldoperationen, darunter fünf in Zentralasien. In dieser Region ist der Europarat nicht vertreten. Überschneidungen der Präsenzen der beiden Organisationen gibt es in Südost- und Osteuropa. Zudem sind die drei Institutionen der OSZE ebenfalls im Feld aktiv.

Die Zusammenarbeit zwischen den OSZE-Feldoperationen und den externen Büros des Europarats ist ungleichmäßig ausgeprägt. In der Ukraine, wo beide Organisationen ihre Präsenz nach 2013/2014 stark ausgebaut haben, ist die Zusammenarbeit relativ weit fortgeschritten. Auf der Grundlage einer Kooperati- onsvereinbarung halten die Organisationen zweimal jährlich gemeinsame Sitzungen $\mathrm{ab}$ und haben ihre Aktivitäten wie auch die Zusammenarbeit mit der EU in einen Aktionsplan integriert. Synergien gibt es etwa bei Reformen des Justizsektors. Der Europarat und der OSZE-Projektkoordinator arbeiten eng bei der Unterstützung der Reform der Generalstaatsanwaltschaft zusammen und die OSZE kann bei der Schulung ukrainischer Richter auf Personal des EGMR zurückgreifen.

Während die Kooperation in der Ukraine ein Positivbeispiel ist, scheint in anderen Ländern eine systematischere Interaktion erforderlich. Dies ist zum Beispiel in Bosnien und Herzegowina der Fall, wo beide Organisationen nahezu identische Prioritäten haben (wie im Aktionsplan des Europarats und im Mandat der OSZE-Mission festgelegt) und mit denselben Partnern und Gebern zusammenarbeiten. Die Zusammenarbeit im Feld ist in denjenigen Ländern schwierig, in denen der politische Kontext sehr sensibel ist oder sich die Dynamik der EU-Integration verlangsamt hat. Ein besonderes Problem entsteht dann, wenn sich die Regierung des Gastlandes von den Aktivitäten einer der Organisationen distanziert hat. Ein solches Verhalten hat vor allem Reputationsgründe - so fürchten die betroffenen Regierungen, durch die Anwesenheit der OSZE mit demokratischen Defiziten oder internen Konflikten in Verbindung gebracht und stigmatisiert zu werden. 


\section{Hindernisse für eine engere Zusammenarbeit}

Seit über einem Jahrzehnt gibt es immer wieder Anstrengungen, um die Zusammenarbeit des Europarats und der OSZE $\mathrm{zu}$ intensivieren. Spezifische Vorschläge dazu enthält das Dokument des Europarats „Beziehungen zwischen dem Europarat und der OSZE: Der Weg nach vorn“ $(2012)^{13}$, der bis dato letzten großen Vorlage zu diesem Thema. Bedauerlicherweise sind aus dem Papier keine konkreten Schritte erwachsen. Als Ursache hierfür nennen Interviewpartner*innen aus Europarat und OSZE die Zurückhaltung auf politischer Ebene und strukturelle Unterschiede zwischen den Organisationen.

\section{Zurückhaltung auf Führungsebene}

In dem Papier von $2012^{14}$ schlug der Europarat unter anderem Prozesse zur regelmäßigen gemeinsamen Entscheidungsfindung und das Wiederaufleben der früher praktizierten gegenseitigen Einladungen vor. Nach einjähriger interner Diskussion reagierte die OSZE Ende 2013 mit Vorsicht und verwies auf die eigene Präferenz für eine „pragmatische, effektive, zielgerichtete, ergebnisorientierte und auf Experten*innen basierende $\mathrm{Zu}$ sammenarbeit", nach Vorstellung der Organisation vor allem ein Austausch zwischen Experten*innen in bestimmten Fällen und im Feld. ${ }^{15}$ Der darauf folgende Vorschlag des belgischen Vorsitzes des Ministerkomitees des Europarats, einen weiteren Konsultationsprozess zwischen den Organisationen hierzu einzuleiten, wurde innerhalb des Europarats nicht ausreichend koordiniert und schließlich nicht zur Diskussion mit der OSZE vorgelegt. ${ }^{16}$ Zwar veranstaltete der belgische Vorsitz noch am Rande der 125. Tagung des Ministerkomitees in Brüssel im Mai 2015 ein hochrangiges außerplanmäßiges Treffen zwischen dem Europarat und der OSZE, seitdem hat jedoch kein weiteres solches Treffen zwischen den Organisationen stattgefunden.

Eine kontroverse Frage ist das sich gegenseitig eingeräumte Rederecht bei den Sitzungen des OSZE-Ministerrates und des Ministerkomitees des Europarats. In der Regel erhalten internationale Organisationen das Wort, nachdem die Vertreter*innen der Mitglieds- beziehungsweise Teilnehmerstaaten gesprochen haben. Im OSZE-Ministerrat hingegen hängt das Rederecht des Generalsekretärs des Europarats von der auf Konsens basierenden Vereinbarung der Teilnehmerstaaten über die Modalitäten des jeweiligen Jahrestreffens ab. Diese Regelung kann dazu führen, dass, wie in Belgrad im Jahr 2015 und in Hamburg im Jahr 2016, aufgrund mangelnder Einigung über die Modalitäten der Sitzungen dem Europarat und anderen internationalen Organisationen gar nicht das Wort erteilt wird. In Belgrad erteilte der serbische amtierende Vorsitzende dem damaligen Generalsekretär des Europarats Thorbjørn Jagland zumindest beim gemeinsamen Mittagessen das Wort. Seit 2016 vertritt der Leiter des Verbindungsbüros des Europarats in Wien die Organisation im OSZE-Ministerrat, allerdings ohne Rederecht. 
Im Mai 2019 sprach OSZE-Generalsekretär Thomas Greminger auf der 129. Sitzung des Ministerkommittees und nahm in diesem Rahmen auch an der Gedenkfeier zum 70. Jahrestag des Europarats teil. Dies war der erste Auftritt nach vier Jahren ohne Teilnahme eines OSZE-Generalsekretärs an dieser turnusmäßigen Sitzung. Bei den Ministersitzungen des Europarats in den Jahren 2018 und 2020 vertrat eine Mitarbeiterin des Büros des Generalsekretärs die OSZE.

Gleichzeitig funktioniert die gegenseitige Vertretung im Ständigen Rat der OSZE und in den Sitzungen der Ministerbeauftragten des Europarats grundsätzlich gut. Wiederholte Anfragen des Europarats nach der Möglichkeit eines dauerhaften Zugangs zum Ständigen Rat blieben jedoch unbeantwortet, da die OSZETeilnehmerstaaten keinen Konsens darüber erzielen konnten. Die im Jahr 2014 vom Schweizer OSZE-Vorsitz eingeleitete Praxis, zu Beginn jeder Sitzung der Entscheidungsgremien Vertreter*innen des Europarats und anderer internationaler Organisationen, die bei der OSZE akkreditiert sind, als Gäste des Vorsitzenden anzukündigen, wird seitdem von jedem Vorsitz fortgesetzt.

Die Bemühungen, häufigere bilaterale Treffen der Generalsekretäre einzuleiten, trugen hingegen erst nach dem Wechsel der Amtsinhaber im Jahr 2017 (OSZE) bzw. 2019 (Europarat) Früchte. So widmeten die Generalsekretäre Greminger und Pejčinović Burić den Beziehungen zwischen ihren Organisationen mehr Aufmerksamkeit und begannen, verschiedene Sitzungsformate wieder aktiv zu nutzen. Die Generalsekretärin des
Europarats sprach im Dezember 2019 und erneut im Dezember 2020 vor dem Ständigen Rat der OSZE.

Die regelmäßigen Zusammenkünfte hochrangiger Mitarbeiter*innen beider Organisationen wurden bereits 2010 eingestellt, da sie als nicht mehr sinnvoll erachtet wurden. Aus Sicht der OSZE waren sie im Grunde durch die Treffen der OSZE-Koordinierungsgruppe überflüssig geworden. Die Leiter der Abteilungen für Auswärtige Beziehungen beider Organisationen treffen sich nach wie vor von Fall zu Fall, um wesentliche Tagesordnungspunkte zu besprechen und Treffen auf hoher Ebene vorzubereiten.

Wiederholt wurde in beiden Organisationen erwogen, dem Dialog in der Koordinierungsgruppe mehr Substanz zu verleihen, insbesondere im Hinblick auf die Erweiterung der bisher auf vier formell vereinbarte Themenbereiche (s.o.) beschränkten Agenda. Problematisch ist dabei, dass aus Straßburger Sicht die OSZE zu wenig Interesse an einer Weiterentwicklung zeigt, wohingegen Wien darauf hinweist, dass diesbezüglich vor allem bei den Teilnehmerstaaten Vorbehalte existieren; diese scheinen zu befürchten, dass die Strukturen zu selbstständig handeln könnten. Andererseits nutzten einige der früheren Vorsitzenden des Ständigen Rats der OSZE und Vorsitzende des Komitees der Ministerbeauftragten des Europarats während ihrer Amtszeit die Gelegenheit, sich in den Sitzungen der Koordinierungsgruppe informell auch zu weiteren Themen auszutauschen und sie so laut Sitzungsteilnehmer*innen $\mathrm{zu}$ „äußerst fruchtbaren Ereignissen“ zu machen. Dies legt nahe, dass statt einer 
Erweiterung des standardisierten Austausches eher ein vermehrter informeller Austausch zielführend wäre.

Insgesamt erschwert die Zurückhaltung bezüglich des Rederechts von Europaratsvertreter*innen bei den OSZEMinistertreffen sowie hinsichtlich einer Erweiterung der thematischen Agenda der Koordinierungsgruppe eine systematischere Kommunikation auf höherer politischer Ebene. Eine effektive Zusammenarbeit zwischen den Organisationen findet daher $\mathrm{zu}$ vielen Themen nur auf den unteren Ebenen zwischen Experten*innen statt.

\section{Strukturelle Unterschiede}

Auch strukturelle Unterschiede stehen einer engeren Zusammenarbeit zwischen den Organisationen im Weg. Die OSZE ist eine Nichtkarriereorganisation mit einem schwachen bürokratischen Apparat, der unter ständiger Beobachtung der Delegationen in Wien steht. Der amtierende Vorsitzende gibt zwar die politische Richtung vor, ist aber letztlich nur der primus inter pares unter den Außenministern. Auch der Generalsekretär hat lediglich ein Mandat als Vertreter des amtierenden Vorsitzes und als Leiter der Verwaltung der Organisation. Ihm obliegt keine direkte Weisungsbefugnis über die drei Institutionen HKNM, ODIHR und RFOM sowie über die 16 Feldoperationen, die alle direkt den kollektiven Entscheidungsgremien der OSZE unterstellt sind. Die Parlamentarische Versammlung der OSZE ist kein integraler Bestandteil der Organisation.
Auch die Befugnisse der Abgeordneten sind nicht mit denen der Parlamentarischen Versammlung des Europarats vergleichbar.

Dagegen spielen im Europarat, einer Karriereorganisation, der Generalsekretär und der bürokratische Apparat eine bedeutende Rolle. Dasselbe gilt für die Abgeordneten und Delegationen der Parlamentarischen Versammlung. Mit dem EGMR wacht zudem ein ständiger und unabhängiger Gerichtshof über die Einhaltung der Normen durch die Mitgliedsstaaten. Die Urteile des Gerichtshofes sind innerhalb dessen gesamter Mitgliedschaft rechtlich bindend und mit wenigen Einschränkungen in der Mehrheit der Mitgliedsstaaten durchsetzbar.

Auch die unterschiedlichen Entscheidungsverfahren tragen zu abweichenden Positionen der Organisationen bei. Die Konsensregel der OSZE reduziert Entscheidungen häufig auf den kleinsten gemeinsamen Nenner. Auch sind Entscheidungen in der OSZE nur politisch bindend und ihre Nichterfüllung kann nicht bestraft werden. Dagegen treffen der Europarat und seine Gremien ihre Entscheidungen, die völkerrechtlich bindenden Charakter haben, nach festgelegten Mehrheitsprinzipien. So können Mitgliedsstaaten überstimmt werden, was beispielsweise bei Russland in Fragen der Ukrainekrise häufig der Fall war. Der Entzug des Stimmrechts Russlands in der Parlamentarischen Versammlung des Europarats nach der Annexion der Krim im Jahr 2014 illustriert die Entscheidungsmechanismen des Europarats. Ein solches Vorgehen wäre in der inklusiven, konsensbasierten OSZE undenkbar. 
Unterschiede gibt es zudem in der Mitglied- bzw. Teilnehmerschaft der Organisationen, auch wenn sie weitgehend identisch sind. Russland ist Mitglied beziehungsweise Teilnehmer beider Organisationen, während Belarus, der Heilige Stuhl, Kanada, die Mongolei und die USA sowie die fünf zentralasiatischen Staaten keine Mitglieder des Europarats sind. ${ }^{17}$ Kanada und die USA hingegen haben im Europarat einen ständigen Sitz als Beobachter und verfolgen die internen Diskussionen der Organisation genau.

Diese organisationsspezifischen Unterschiede erschweren die Zusammenarbeit zwischen beiden Organisationen. Unterschiedliche Grade der Verbindlichkeit getroffener Vereinbarungen, unterschiedliche Entscheidungsverfahren und Varianz bei der institutionellen Autonomie stellen Hindernisse dar, um auf politischer Ebene zu gemeinsamen Positionen in sensiblen Fragen zu gelangen.

\section{Empfehlungen}

Eine engere Zusammenarbeit zwischen Europarat und OSZE erfordert mehr als eine rhetorische Selbstverpflichtung der Regierungen, multilaterale Plattformen zu nutzen. Gerade die größeren Staaten sollten in beiden Organisationen aktiver sein. Dies gilt nicht nur, aber insbesondere, für die größeren EU-Mitgliedsstaaten und die Institutionen der EU. Für die EU sind sowohl der Europarat (z. B. in Beitrittsprozessen) als auch die OSZE (z. B. in Konflikten wie in der Ostukraine oder in Regionen, in denen die EU nur über begrenzten Einfluss verfügt) wertvolle Partner. Die zentrale Herausforderung ist dabei, bestehende formale Kooperationsvereinbarungen mit Leben zu füllen. Die Berichterstattergruppe für Auswärtige Beziehungen des Europarats brachte diese Idee im Jahr 2012 auf den Punkt: „Es besteht wahrscheinlich kein großer Bedarf an zusätzlichen, verbindlichen Konventionen. Der Schwerpunkt sollte auf der Umsetzung liegen. "18 Nachfolgend werden daher praktische Schritte für eine engere Zusammenarbeit aufgezeigt.

\section{Verbesserung der Kommunikation auf Führungsebene}

Auf der Ebene hochrangiger Mitarbeiter*innen und der politischen Führung ist die Kommunikation zwischen den Organisationen übermäßig ritualisiert. Auch thematisch gibt es auf dieser Ebene wenig Flexibilität. Es ist den Regierungen daher zu raten, eine Verbesserung der Kommunikation zwischen den Organisationen auf politischer Ebene anzustreben. Ein erster Schritt in diese Richtung könnte darin bestehen, den Diskussionen auf der halbjährlichen Zusammenkunft der Koordinierungsgruppe mehr Substanz zu verleihen. Da eine Änderung der formalen Modalitäten bei den Treffen bisher unrealistisch ist, sollte die Möglichkeit in Betracht gezogen werden, die Diskussionen zunächst durch informelle Konsultationen zu erweitern.

- Erstens sollten die beiden Organisationen erwägen, mehr Gewicht auf Expertenbesprechungen am Rande der 
Treffen der Koordinierungsgruppe zu legen. Vertreter*innen beider Organisationen haben von positiven Auswirkungen informeller Beratungen berichtet. Bei diesen Treffen könnten Themen behandelt werden, die bisher nicht Gegenstand der vier formalen Tagesordnungspunkte der Koordinierungsgruppe sind. Exemplarisch sind hier zu nennen: Migration, demokratische Sicherheit, Nichtdiskriminierung, Jugend und Sicherheit, transnationale Risiken, Medienfreiheit, Sicherheit von Journalisten und neue Technologien (einschließlich künstliche Intelligenz sowie deren Einfluss auf Demokratie und Menschenrechte). Aufgrund ihrer informellen Ausgestaltung böten diese Treffen zugleich eine Gelegenheit, auch aktuelle Themen wie Reaktionen auf die COVID-19-Pandemie und Feldoperationen zu erörtern.

- Zweitens wäre zu erwägen, ob die Koordinierungsgruppe unter dem Tagesordnungspunkt "Sonstiges" mit der Ausarbeitung gemeinsamer thematischer Prioritäten und Standpunkte beauftragt werden könnte, um Treffen mit den Mitglieds- bzw. Teilnehmerstaaten vorzubereiten.

- Drittens sollten, wie im „Gemeinsamen Katalog" von 2000 bereits vorgesehen ${ }^{19}$, die "2+2"-Treffen auf Führungsebene wiederbelebt werden. Als OSZE-Vorsitz könnte Schweden im Jahr 2021 mit Unterstützung der Troika-Mitglieder Albanien und Polen sowie der drei Vorsitze des Ministerkomitees des Europarats (Deutschland, Ungarn und Italien) die Initiative er- greifen, diese Treffen wieder aufzunehmen.

\section{Unterstützung der Feldkooperation}

Obwohl die Zahl der vom Europarat und der OSZE unterhaltenen Feldpräsenzen ähnlich ist, ist das Gewicht, das sie in den beiden Organisationen haben, unterschiedlich. Der operative Schwerpunkt des Europarats liegt in seinem Hauptquartier, während die OSZE mehr als drei Viertel ihres Personals im Feld hat. Der Europarat mit seinen Konventionen, seiner Rechtsexpertise, seinen Monitoring-Organen und seiner soliden finanziellen Ausstattung ist ein wertvolles Pendant zur OSZE mit ihren schnellen Reaktionskapazitäten und ihrem Know-how sowie ihren Aktivitäten in Bereichen wie Konfliktmanagement und Demokratisierung.

Die sich ergänzenden Charakteristika der beiden Organisationen sowie deren ähnliche Zielsetzungen eröffnen viele Koordinierungs- und Kooperationsmöglichkeiten im Feld. Daher ist eine systematischere, fallbezogene und auf die Bedürfnisse der einzelnen Gastländer zugeschnittene Zusammenarbeit zwischen der OSZE und dem Europarat auch kein völlig neuer Vorschlag. ${ }^{20}$ Die Zusammenarbeit beispielsweise in der Ukraine ist vergleichsweise systematisch organisiert. Hierauf kann man aufbauen. Daraus folgt:

- Erstens sollten die Regierungen eine stärkere Interaktion zwischen der OSZE und dem Europarat in den 
Ländern fördern und finanzieren, in denen beide Organisationen Feldoperationen bzw. externe Büros unterhalten. Synergieeffekte könnten sich aus der Kombination von lokalem Fachwissen und Personal der OSZE mit dem juristischen Fachwissen und den finanziellen Ressourcen des Europarates ergeben.

- Zweitens sollte die EU ihren Einfluss in beiden Organisationen nutzen, um die Interaktion zwischen OSZE und Europarat im Feld zu stärken. Die EU verbindet eine besondere Partnerschaft mit dem Europarat, die auf einem „Rahmen für verstärkte Zusammenarbeit und politischen Dialog“" und einer Reihe von Vereinbarungen und Arbeitsplänen basiert. ${ }^{21}$ Darüber hinaus wurden bereits Schritte unternommen, um enger mit der OSZE zusammenzuarbeiten. ${ }^{22}$ Die Präsidentschaften respektive Vorsitze der EU, des Europarats und der OSZE könnten zusammen eine politische Initiative starten, um die Stärkung der gemeinsamen Feldarbeit zu erörtern.

- Drittens sollten beide Organisationen sicherstellen, dass ihre politische und rechtliche Beratung kompatibel ist. Hierzu sollte die OSZE häufiger von der Rechtssprache des Europarats Gebrauch machen. Im Gegenzug könnte der Europarat die politischen Empfehlungen der OSZE in den Bereichen demokratische Wahlen, Schutz der fundamentalen Menschenrechte, Rechtsstaatlichkeit sowie Toleranz und Nichtdiskriminierung systematischer nutzen. Letztlich wird eine bessere Abstimmung ihrer Bera- tung die Legitimität beider Organisationen und ihre politische Wirkung im Feld erhöhen.

\section{Notizen}

1 Die Autoren danken den Interviewpartnern sowie den Gutachtern für ihre vielen wertvollen Kommentare.

2 Der Europarat hat ein jährliches Budget von 496 Mio. $€$ zuzüglich freiwilliger $\mathrm{Zu}$ schüsse und über 2.500 Mitarbeiter, während die OSZE ein jährliches Budget von ungefähr 139 Mio. $€$ zuzüglich extra-budgetärer Mittel und 2.880 Mitarbeiter hat. Siehe: „The Council of Europe in brief: Budget", Council of Europe, https://ww w.coe.int/en/web/about-us/budget?deskto $\mathrm{p}=$ true; OSCE, Approval of the 2020 Unified Budget, PC.DEC/1369, 28 May 2020, https://www.osce.org/permanent-council /453804; ,Who we are: Funding and budget", OSCE, https://www.osce.org/fundin g-and-budget.

3 CoE/OSCE, Relations Between the Council of Europe and the OSCE: Common Catalogue of Co-operation Modalities, SEC.GAL/30/00, 4 April 2000 und $\mathrm{CM}(2000) 52,25$ April 2000, https://rm.c oe.int/CoERMPublicCommonSearchSer vices/DisplayDCTMContent?documentI $\mathrm{d}=0900001680599 \mathrm{~d} 8 \mathrm{~d}$.

4 OSCE, Enhanced Co-operation Between the Organization for Security and Co-operation in Europe (OSCE) and the Council of Europe (CoE), PC.DEC/637, 2 December 2004, https://www.osce.org/pc/14 732.

5 Ebd.

6 Das letzte Treffen dieser Art fand am Rande der UN-Generalversammlung in New York am 21. September 2011 statt und wurde vom litauischen OSZE-Vorsitz veranstaltet. $\mathrm{Zu}$ diesem Zeitpunkt war der Europarat als Veranstalter an der 
Reihe, allerdings konnten die Vorsitze von 2012-14 weder Zeit noch Ort für ein "2+2"-Treffen finden. Ein weiteres hochrangiges Treffen im "2+3"-Format (inklusive des bevorstehenden Vorsitzes von Bosnien und Herzegowina) fand im Jahr 2015 unter dem belgischen Vorsitz in Brüssel statt, blieb aber seitdem das letzte seiner Art.

7 CoE/OSCE, oben zitiert (Anm. 3).

8 OSCE, oben zitiert (Anm. 4).

9 CoE/OSCE, Declaration on Co-operation between the Organization for Security and Co-operation in Europe and the Council of Europe, 17 May 2005, https:// rm.coe.int/CoERMPublicCommonSearc hServices/DisplayDCTMContent?docum entId=090000168046a481.

10 CoE Directorate of External Relations, OSCE: Background Information, 6 November 2017.

11 Siehe „Fundamental rights“, Council of Europe, Venice Commission, https:/ww w.venice.coe.int/WebForms/pages/?p=02 _Rights\&lang=EN.

12 Diese Themenfelder schließen Toleranz und Nicht-Diskriminierung, Migration, Rechtsstaatlichkeit, demokratische Regierungsführung, Sinti und Roma-Themen, Geschlechtergleichberechtigung sowie die Kommunikation von demokratischen Werten und Menschenrechten ein.

13 CoE Ministers' Deputies/Rapporteur Groups, Relations Between the Council of Europe and with the OSCE: The Way Forward, GR-EXT(2012)11-rev, 25 October 2012, https://search.coe.int/cm/Pages/ result_details.aspx?ObjectID $=0900001680$ $5 \mathrm{c} 9 \mathrm{~b} 83$.

14 Ebd.

15 Igor Prokopchuk, Chairperson of the OSCE Permanent Council, Letter to the Permanent Representative of Austria to the Council of Europe/Chairperson of the Council of Europe's Committee of
Ministers' Deputies, CIO.GAL/186/13, 30 December 2013.

16 Belgian Chair of the CoE's Committee of Ministers, Possibilities for enhanced cooperation between the Council of Europe $(\mathrm{CoE})$ and the Organization for Security and Co-operation in Europe (OSCE), January 2015.

17 Der Europarat unterhält konstruktive Beziehungen zu den fünf zentralasiatischen Staaten. Kasachstan und Kirgisistan sind Teil der 2011 entworfenen Politik des Europarats für Nachbarschaftsregionen. 2007 haben sich die Venedig-Kommission und die zentralasiatischen Staaten auf eine Zusammenarbeit geeinigt, wobei entsprechende Projekte durch die EU und einige Mitgliedsstaaten des Europarats finanziert werden. Eine gemeinsame Übereinkunft zum Zentralasiatischen Rechtsstaatlichkeitsprogramm wurde mit allen fünf Staaten im Januar 2020 getroffen. Kasachstan schloss sich zudem im selben Monat der Gruppe von Staaten gegen Korruption des Europarats (GRECO) an.

18 CoE Secretariat General, Rapporteur Group on External Relations, Enhancing Co-operation and Complementarity Through Greater Coherence, DD(2012)40, 16 January 2012. Dieser Fortschrittsbericht bezieht sich auf den Ulfstein-Report (DPP (2012)1, 23 March 2012), später unter demselben Titel publiziert, https://rm.coe.int/CoERMPublic CommonSearchServices/DisplayDCTMC ontent?documentId=0900001680599dd7.

19 CoE/OSCE, oben zitiert (Anm. 3).

20 Siehe z.B.: Igor Prokopchuk, Chairperson of the OSCE Permanent Council, oben zitiert (Anm. 15).

21 Siehe: Memorandum of Understanding between the Council of Europe and the European Union, 11 and 23 May 2007, https://rm.coe.int/1680597b32. Die EU hat bisher einige hundert EU-Europaratsprogramme gefördert. 
22 Ein Grundsatzbekenntnis zu einer verstärkten Kooperation zwischen der EU und der OSZE findet sich in der Korrespondenz zwischen der Hohen Repräsen- tantin der EU Federica Mogherini und OSZE Generalsekretär Thomas Greminger vom 22. Juni 2018. 